\title{
UNDERSTANDING THE RELATIONSHIP BETWEEN GAS AND ICE: EXPERIMENTAL INVESTIGATIONS ON ORTHO-PARA RATIOS
}

\author{
J.-H. Fillion ${ }^{1,2}$, M. Bertin ${ }^{1,2}$, A. Lekic ${ }^{1,2}$, A. Moudens ${ }^{1,2}$, L. Philippe ${ }^{1,2}$ \\ and X. Michaut ${ }^{1,2}$
}

\begin{abstract}
Numerous hydrogenated molecules observed in comets and the interstellar medium present nuclear spin isomers. The non-thermal ortho: para ratios measured in space are believed to be of high diagnostic value, allowing remote measurement of physical conditions prevailing in the past, such as the initial temperature associated with a molecular formation or condensation. The lack of laboratory studies on interactions at the gas-solid interface limits our current interpretation of observations. Recent progress in nuclear spin conversion (NSC) dynamics are discussed in this paper. Results on water and methane in cryogenic matrices illustrate the conversion mechanisms within the condensed phase at very low temperatures. NSC of molecular hydrogen at the surface of $10 \mathrm{~K}$ Amorphous Solid Water (ASW) shows surface mediated conversion processes for molecular hydrogen. NSC involving molecular ices are discussed in the light of few recent studies, including UV photodesorption investigations.
\end{abstract}

\section{Introduction}

Molecules with identical nuclei having non-zero spin are known to exist in different nuclear spin modifications (ortho-para, A-E...) that are also called "nuclear spin isomers". A well-known example is molecular hydrogen $\mathrm{H}_{2}(\mathrm{H}$ nuclei having a $1 / 2$ spin) that exists as ortho and para isomers, depending on whether the spins of the protons are parallel (total nuclear spin $\mathrm{I}=1$ ) or anti-parallel (total nuclear spin $\mathrm{I}=0)$, respectively. Spin isomers also exist for many other molecules $\left(\mathrm{H}_{2} \mathrm{O}\right.$, $\mathrm{H}_{2} \mathrm{CO}, \mathrm{NH}_{3}, \mathrm{CH}_{4}$..) that are observed in the interstellar medium (Lis et al. 2010) and/or cometary atmospheres (Shinnaka et al. 2011). The spin isomers ratio (or "ortho-to-para ratio") obtained through the analysis of rotational lines can be

${ }^{1}$ UPMC Université Paris 06, UMR 7092, LPMAA, 75005 Paris, France

2 CNRS, UMR 7092, LPMAA, 75005 Paris, France 
also expressed by the "spin temperature" $\mathrm{T}_{\text {spin }}$ in which a Boltzmann distribution reproduces the ratio of the spin isomers. $\mathrm{T}_{\text {spin }}$ values observed in space vary substantially from the kinetic or rotational temperatures. Although the precise origin of this non-equilibrium is not well understood, it has been suggested that the spin temperature could trace the chemical and/or physical histories of the molecules (Lis et al. 2010).

In the case of $\mathrm{H}_{2}$, the spin temperature observed in protostellar environments has been interpreted as a fossil record of the thermal history of the interstellar gas, characterizing the epoch before the gas temperature was elevated by the passage of a shock (Neufeld et al. 2006). This "fossil" character is supported by the fact that the spin temperature can be preserved for long timescales in the gas phase, since conversion between spin isomers is extremely slow by radiative transition (Pachucki et al. 2008) and through non-reactive collisions (Tudorie et al. 2006). Ortho-para transitions can be driven by reactive collision with $\mathrm{H}, \mathrm{H}^{+}$(and $\mathrm{H}_{3}^{+}$) in some stellar environments (Flower et al. 2006; Honvault et al. 2011). Chemical conversion channels require long time scales to reach a steady state regime (Pagani et al. 2009, 2010). NSC of $\mathrm{H}_{2} \mathrm{CO}$ induced by non-reactive collisions has also been investigated theoretically for $\mathrm{H}_{2}$ densities and temperatures relevant to protostellar environments (Tudorie et al. 2006). The derived conversion time was much longer than the lifetime of $\mathrm{H}_{2} \mathrm{CO}$, leading to the conclusion that non-reactive conversion channels can be neglected in most interstellar conditions.

The fact that nuclear spin relaxation can be very slow in many circumstances has encouraged some authors to assign the spin temperature to a primordial temperature, that could indicate the initial physical conditions for the formation of the molecules. Spin temperatures determined in the coma of solar-systems comets mostly lie in the 20-40 K range for $\mathrm{H}_{2} \mathrm{O}$ and $\mathrm{NH}_{3}$ typically (Bonev et al. 2007; Bockelée-Morvan et al. 2009; Shinnaka et al. 2011). Similar spin temperatures were also observed for $\mathrm{CH}_{4}$ in some comets (Kawakita et al. 2005). These temperatures coincide neither with the temperature of the ice, nor with the temperature of the surface. A common spin-temperature for any comets and any molecule supports the interpretation of the spin temperature as the physical temperature of the grain on which the ice has been formed (Kawakita et al. 2005; Shinnaka et al. 2011). The underlying hypothesis is that $\mathrm{T}_{\text {spin }}$ in cometary ices is preserved over the time of 4.5 billion years, during which comets are mostly at $50 \mathrm{~K}$ or less, then heated to temperatures higher than $180 \mathrm{~K}$, at which they sublimate. Interestingly, the recent detection of the emission lines from cold water vapor from the disk around the young star TW Hydrae yields to a lower spin temperature $(\mathrm{T}=13.5 \mathrm{~K})$ as compared to cometary water. This suggests a very low formation temperature for these cold ice-coated pre-cometary grains (Hogerheijde 2011).

We point out that the interpretation of the spin temperatures of molecules formed or condensed on grains is puzzling, since very little is known concerning the relaxation of spin isomers in cold solids, on surfaces of astrophysical interest and after thermal/non-thermal desorption (ice sublimation, photodesorption or chemistry induced desorption, see Collings and McCoustra in this volume). Though the relaxation of the spin isomers can be very slow in some pure gas 


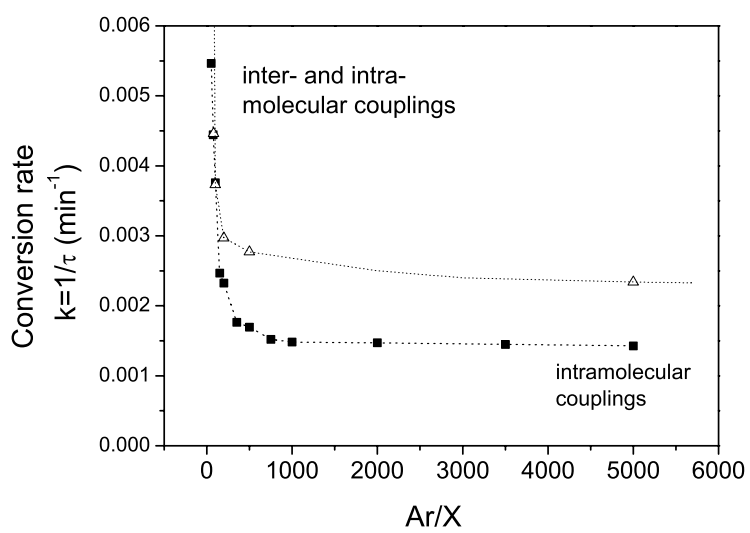

Fig. 1. Evolution of conversion rate versus dilution of molecule $\mathrm{X}$ isolated in an argon matrix at $4.2 \mathrm{~K}$. Water (full squares), methane (open triangles).

phase systems, it should not be generalized to any species for any temperatures and densities. In the following, we review some recent results and current studies that could help the interpretation in astrophysical context, with emphasis on some experimental approaches.

\section{Experiments in cold matrices}

Experiments in rare gas matrices are very convenient for investigating parameters governing nuclear spin relaxation for isolated molecules at very low temperatures. Pardanaud and coworkers have studied the evolution of the conversion rate as a function of the dilution of water in the sample at $4.2 \mathrm{~K}$ (Pardanaud 2007). For dilution of water in argon higher than 2000, a slow conversion time of typically 700 minutes is observed independent of the water concentration (Pardanaud 2007). This slow conversion rate is due to "intrinsic" interaction of the molecule with the matrix and intra-molecular magnetic couplings (Cacciani et al. 2012). When the concentration of water is increased (dilution below 2000) an additional inter-molecular mechanism is taking place: a water molecule interacts with the inhomogeneous magnetic field produced by the two protons of a neighboring molecule (Pardanaud 2007). In this "high concentration" regime, conversion times of less than 200 minutes have been for example measured for water trapped in argon at $4.2 \mathrm{~K}\left(\mathrm{H}_{2} \mathrm{O} / \mathrm{Ar}=50\right)$. Experiments performed by Lekic et al. show the same behavior with concentration for $\mathrm{CH}_{4}$, demonstrating the generality of the two regimes driven by inter- and intra-molecular interactions, as shown in Figure 2 (Lekic 2011). These experiments strongly suggest that very short conversion times can be expected in solid phase where the molecular concentration is far higher that produced in matrices. In another series of experiments involving $\mathrm{CH}_{4}$ trapped in argon matrices, the role of the temperature on the NSC dynamics has 

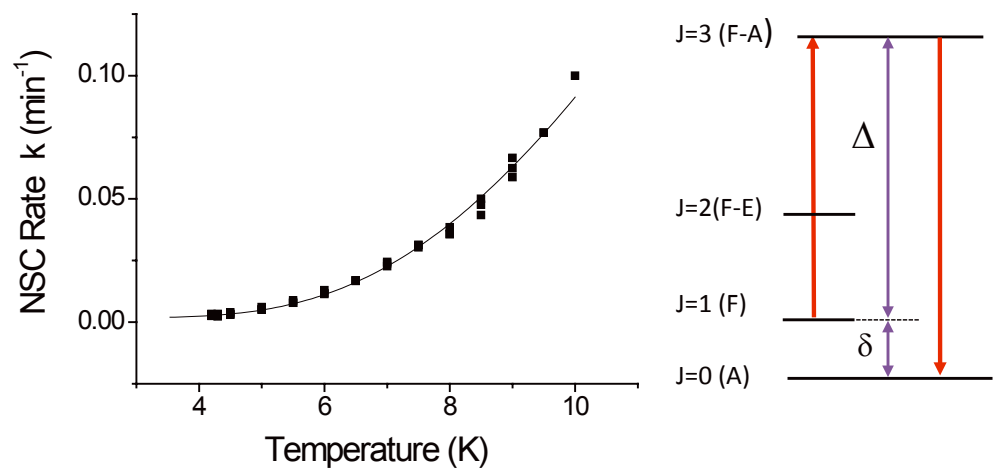

Fig. 2. Left: NSC rates of $\mathrm{CH}_{4}$ in Argon in the high dilution regime $\left(\mathrm{Ar} / \mathrm{CH}_{4}=5000\right)$. Experimental value (squares); model $k=A+B \exp (-34,6 / T)+C T^{6,27}$ (full line). Right: energetic diagram illustrating the two-phonon resonant process in $\mathrm{CH}_{4}$ (Orbach process).

been investigated. The results obtained in the high dilution regime ("intrinsic" relaxation mechanism) are presented in Figure 2. From 4 to $10 \mathrm{~K}$, the conversion rates increase by more than one order of magnitude, with typical conversion times reducing from 400 minutes at $4 \mathrm{~K}$ to 10 minutes at $10 \mathrm{~K}$. The evolution of the conversion rates with temperature can be reproduced considering several relaxation mechanisms for the rotational energy (Lekic 2011). The dominating contribution to the conversion rate $\mathrm{k}$ is given by $k \propto \exp (-\Delta / k t)$, where the energy gap $\Delta$ was found to match the energy difference between the $\mathrm{J}=1$ and $\mathrm{J}=3$ rotational levels of $\mathrm{CH}_{4}$ in the argon cage. Considering the role of phonons in the processes, this is consistent with a two-stage resonant process involving the absorption of one phonon of energy delta, followed by the emission of a second photon with energy $\Delta+\delta$, also known as an Orbach process (Lekic 2011; Scott \& Jeffries 1962). In the present case, $\delta$ is the energy relaxation from ortho- $\mathrm{CH}_{4}(\mathrm{~J}=1)$ to meta- $\mathrm{CH}_{4}$ $(\mathrm{J}=0)$ (Fig. 2). This experiment shows that conversion rates can accelerate dramatically with temperature. The origin of this enhancement is the relaxation of the rotational energy via a resonant process involving the coupling with the matrix, demonstrating the important role played by both energy relaxation and the molecular environment in the dynamics of NSC.

\section{Experiments on cold surfaces}

Experiments involving molecules on surfaces have been in the past mainly focused on metallic surfaces, but detailed investigations of the nuclear spin conversion of molecular hydrogen adsorbed on Amorphous Solid water (ASW) have been recently explored (Chehrouri 2011; Sugimoto \& Fukutani 2011). In these experiments, ASW film maintained at $10 \mathrm{~K}$ is exposed to a sub-monolayer of normalroom temperature ortho-to-para ratio $\left(\mathrm{T}_{\text {spin }}=300 \mathrm{~K}\right)$ of a molecular hydrogen (or deuterium) beam. After a delay, the sample is heated from 10 to $30 \mathrm{~K}$ with a 


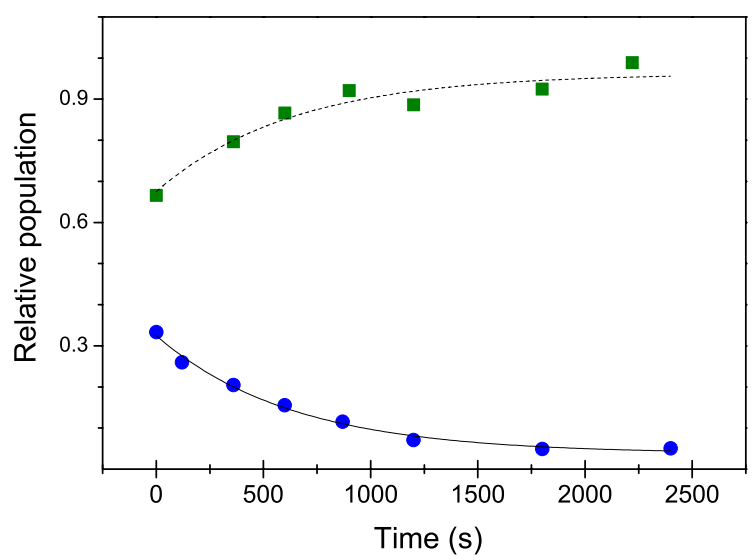

Fig. 3. Time evolution of the J-selected relative populations of $0.75 \mathrm{ML}$ molecular $\mathrm{D}_{2}$ on porous ASW with $0.012 \mathrm{ML}$ of co-adsorbed $\mathrm{O}_{2}$; para- $\mathrm{D}_{2}(\mathrm{~J}=1)$ (full circles) and ortho- $\mathrm{D}_{2}(\mathrm{~J}=0)$ populations (full squares); from Chehrouri (2011).

linear $\operatorname{ramp}(10 \mathrm{~K} / \mathrm{min})$. The desorption signal is recorded by detecting ions produced by a laser focused just in front of the surface. The wavelength of the laser is tuned to ionize selectively either ortho or para isomers by selecting a specific $\mathrm{Q}(\mathrm{J})$ transition of the $(2+1)$ REMPI $(\mathrm{E}, \mathrm{F} \leftarrow X)$ spectrum. Exploration of the spectrum reveals only two rotational lines with significant intensities, corresponding to the two lowest rotational energy levels $\mathrm{J}=0$ and $\mathrm{J}=1$, as expected from Boltzmann distribution at low temperature $(10-30 \mathrm{~K})$. This indicates that the molecules from the initial $300 \mathrm{~K}$ molecular beam have been efficiently thermalized on the cold surface. By contrast, the spin temperature has kept its initial value $(300 \mathrm{~K})$. One can conclude that thermal equilibrium of the gas adsorbed on the surface was achieved, but that no spin conversion had occurred on the time scale of the experiment (a few minutes), neither due to the interaction of $\mathrm{D}_{2}$ with the $10 \mathrm{~K}$ ASW surface, nor due to the heating ramp (10 to $30 \mathrm{~K})$. Experiments with longer residence times were necessary to observe NSC. As an example, we present in Figure 3 the results obtained for $\mathrm{H}_{2}\left(\mathrm{D}_{2}\right)$ in the presence of $\mathrm{O}_{2}$ co-adsorbed on the surface. The population of $\operatorname{para}-\mathrm{D}_{2}(\mathrm{~J}=1)$ is clearly decreasing following a single exponential decay with a decay time constant of $600 \mathrm{~s}$, while that of ortho$\mathrm{D}_{2}(\mathrm{~J}=0)$ is increasing with the same dynamics (Fig. 3a), showing the progressive nuclear spin conversion process. By varying the amount of molecular oxygen adsorbed on the surface, one can control the conversion time. This behavior can be qualitatively explained considering that molecular hydrogen present on surface can diffuse and experience the magnetic field created close to the molecular oxygen centres. The presence of a tiny amount of molecular oxygen, as low as $0.1 \%$ of total molecular concentration on the surface, was found sufficient to significantly increase the conversion rates (Chehrouri et al. 2011). We estimate the conversion rate to be below $15 \%$ per hour on an oxygen free surface. This rate is three orders of magnitude larger than that found in pure solid hydrogen. To explain this effect, 
Sugimoto \& Fukutani argue that giant electric fields with a steep atomic-scale gradient present on ASW surface can induce significant Stark effect and spin-orbit couplings, and propose an interesting mechanism that mixes ortho-para states on the surface (Sugimoto \& Fukutani 2011). We can conclude that NSC of molecular hydrogen under interstellar conditions depends on the effective residence time of molecules on the surface and is expected to be efficient in dark clouds.

\section{Discussion}

The experiments discussed above demonstrate that whatever the origin of the magnetic couplings may be (intra- or inter- molecular), NSC can be strongly enhanced by several external parameters (temperature, molecular impurities, strong electric surface field etc.). Though experiments in matrices are convenient to probe the role of a specific parameter, one can put forward these systems to be not very relevant for astrophysics. It should be noted that in matrices, as well as in the case of sub-monolayer of $\mathrm{H}_{2}\left(\mathrm{D}_{2}\right)$ adsorbed on the top of a $10 \mathrm{~K}$ ASW surface, molecules are almost freely rotating. These laboratory conditions are not so different from gas phase conditions. In solid water ice on the contrary, hydrogen bonding and other intermolecular interactions act as a hindering potential for the free rotation of the water molecule, quenching the energy splitting between ortho and para states toward extremely small values. The electric interactions cause the presence of a rotational barrier between spin isomers, which governs the conversion rates via quantum tunneling. Buntkowsky et al. have modeled the conversion of para- $\mathrm{H}_{2} \mathrm{O}$ to ortho- $\mathrm{H}_{2} \mathrm{O}$ in a four spin system under the influence of homonuclear magnetic dipolar interactions and spin-spin couplings (Buntkowsky et al. 2008). This study shows in particular that in the case of evaporation, the spin conversion depends strongly on the speed at which the region of rotational hindering is passed. This calculation clearly suggests that the detail of the evaporation process is crucial to explain the effective spin temperature when going from solid to gas phase and conversely, as pictured in Figure 4. Pure para $-\mathrm{H}_{2} \mathrm{O}$ generated in $\mathrm{Ar}$ matrices, followed by subsequent fast annealing generating ice particles indicate fast reconversion of nuclear spin to equilibrium, with fast NSC occurring in water dimers and clusters (Sliter 2011). Spin relaxation has also been observed in some circumstances in supersonic expansion, where water clusters are plausibly involved as intermediates for NSC (Manca et al. 2011). In the case of thermal desorption, new experiments reveal that the nuclear-spin temperature is not sensitive to the thermal or chemical history of the water ice sample, but lies systematically in the high temperature limit. This demonstrates that no information on the surface temperature at which $\mathrm{H}_{2} \mathrm{O}$ molecules condensed or formed can be retrieved through spin temperature measurement in the gas phase and after a desorption step (Hama et al. 2011).

\section{Conclusion}

Effort has still to be made to interpret the nuclear-spin temperatures of molecules observed in interstellar space or cometary comae, since no observation of low 


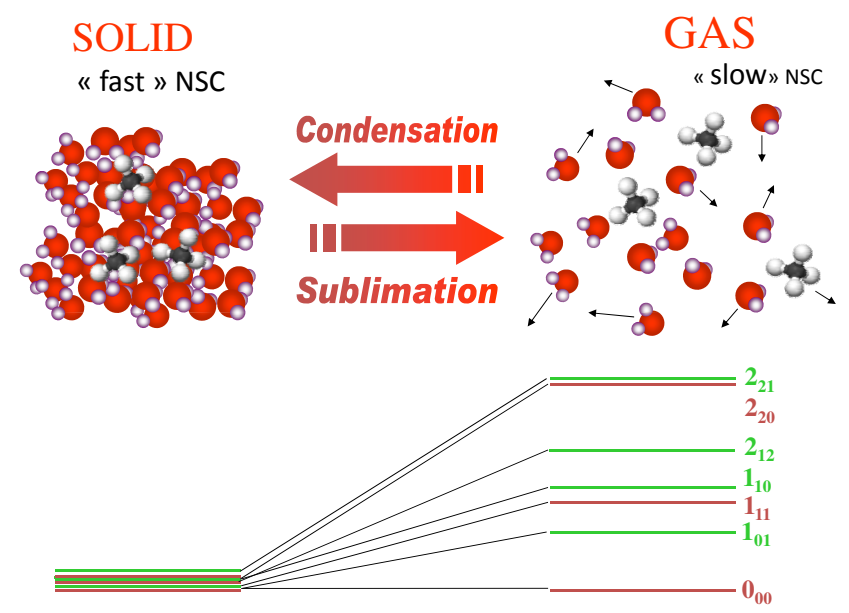

Fig. 4. Picture of gas-solid interplay and NSC dynamics.

spin-temperature after desorption has been ever reported in laboratory conditions. To this end, one should compare "sudden" and slow desorption respectively. The mechanisms involved in the photodesorption process are in this context very important to consider. Recent wavelength dependent studies (Fayolle et al. 2011) demonstrate that Desorption Induced by Electronic Transition (DIET) is responsible for the desorption of CO from the top-most layers of the CO ice. In contrast to this direct mechanism, the UV photodesorption of pure water ice is predicted to be rather indirect, starting with the photodissociation of water, followed by energetic $\mathrm{H}$ atoms kicking out a neighboring molecule or by desorption induced by $\mathrm{H}$ and $\mathrm{OH}$ recombination (Andersson \& van Discoeck 2008). Photodesorption experiments conducted at $157 \mathrm{~nm}$ on polycrystalline water $\left(\mathrm{H}_{2} \mathrm{O}\right.$ and $\left.\mathrm{D}_{2} \mathrm{O}\right)$ provide evidence for the kick-out mechanism (Hama 2010). Such experiments should be performed at lower temperatures to further explore the spin temperature of water after UV photodesorption. New experimental and theoretical approaches are desired to tackle the interpretation of ortho-to-para ratios in space.

The authors would like to acknowledge the strong financial supports from the Agence Nationale de la Recherche (ANR) under contract ANR-09-BLAN-0066-01 and the national program PCMI (Physique et Chimie du Milieu Interstellaire) funded by the CNRS.

\section{References}

Andersson, S., \& van Dishoeck, E.F., 2008, A\&A, 491, 907

Bonev, B.P., Mumma, M.J., Villanueva, G.L., et al., 2007, ApJ, 661, L97

Bockelée-Morvan, D., Woodward, C.E., Kelley, M.S., \& Wooden, D.H., 2009, ApJ, 696, 1075

Buntkowsky, G., Limbach, H.H., Walaszek, B., et al., 2008, Z. Phys. Chem.-Int. JRPCCP, 222, 1049 
Cacciani, P., Cosleou, J., \& Khelkhal, M., 2012, PRA, accepted

Chehrouri, M., Fillion, J.H., Chaabouni, H., et al., 2011, PCCP, 13, 2172

Crovisier, J., 2006, Mol. Phys., 104, 2737

Fayolle, E.C., Bertin, M., Romanzin, C., et al., 2011, ApJ, 739, L36

Hama, T., Watanabe, N., Kouchi, A., \& Yokoyama, M., 2011, ApJ, 738, L15

Hogerheijde, M.R., Bergin, E.A., Brinch, C., et al., 2011, Science, 334, 338

Honvault, P., Jorfi, M., Gonzalez-Lezana, T., Faure, A., \& Pagani, L., 2011, PRL, 107, 023201

Kawakita, H., Dello Russo, N., Furusho, R., et al., 2006, ApJ, 643, 1337

Lekic, A., 2011, Ph.D. Thesis, Univ Pierre \& Marie Curie, France

Lis, D.C., Phillips, T.G., Goldsmith, P.F., et al., 2010, 65 coauthors, A\&A, 521, L26

Manca, T., Quack, M., \& Schmidiger, 2011, Faraday Discuss., 150, 120

Pachucki, K., \& Komasa, J., 2008, PRA, 77, 030501

Pagani, L., Roueff, E., \& Lesaffre, P., 2011, ApJ, 739, L35

Pardanaud, C., 2007, Ph.D. Thesis, Univ Pierre \& Marie Curie, France

Scott, P.L., \& Jeffries, C.D., 1962, PR, 127, 32

Shinnaka, Y., Kawakita, H., Kobayashi, H., et al., 2011, ApJ, 729, 81

Sliter, R., Gish, M., \& Vilesov, A.F., 2011, JPCA, 115, 9682

Sugimoto, T., \& Fukutani, K., 2011, Nat. Phys., 7, 307

Tudorie, M., Cacciani, P., Cosleou, J., et al., 2006, A\&A, 453, 755 